\title{
Draft Genome Assembly and Annotation of Red Raspberry Rubus Idaeus
}

Running title: The red raspberry genome sequencing

Haley Wight ${ }^{1,2}$, Junhui Zhou ${ }^{1}$, Muzi Li ${ }^{1,2}$, Sridhar Hannenhalli ${ }^{1,2}$, Stephen M. Mount ${ }^{1,2}$ and Zhongchi Liu ${ }^{1 *}$

1.Dept. of Cell Biology and Molecular Genetics, University of Maryland, College Park, MD 20742

2.Center for Bioinformatics and Computational Biology, University of Maryland, College Park, MD20742

Emails: haleywight18@gmail.com, junhui55@umd.edu, limuzi92@ terpmail.umd.edu, sridhar@umiacs.umd.edu, smount@umd.edu, zliu@umd.edu*

\section{*Corresponding author}

Zhongchi Liu

Dept. of Cell Biology and Molecular Genetics

University of Maryland, College Park, MD 20742

Tel: 301-405-1586

Fax: 301-314-9489

zliu@umd.edu 


\section{Abstract}

2 The red raspberry, Rubus idaeus, is widely distributed in all temperate regions of Europe, Asia,

3 and North America and is a major commercial fruit valued for its taste, high antioxidant and

4 vitamin content. However, Rubus breeding is a long and slow process hampered by limited

5 genomic and molecular resources. Genomic resources such as a complete genome sequencing

6 and transcriptome will be of exceptional value to improve research and breeding of this high

7 value crop. Using a hybrid sequence assembly approach including data from both long and short

8 sequence reads, we present the first assembly of the Rubus idaeus genome (Joan J. variety). The

9 de novo assembled genome consists of 2,145 scaffolds with a genome completeness of $95.3 \%$

10 and an N50 score of $638 \mathrm{~KB}$. Leveraging a linkage map, we anchored $80.1 \%$ of the genome onto

11 seven chromosomes. Using over 1 billion paired-end RNAseq reads, we annotated 35,566

12 protein coding genes with a transcriptome completeness score of 97.2\%. The Rubus idaeus

13 genome provides an important new resource for researchers and breeders.

14

15 Key words: red raspberry, genome assembly, annotation, genome comparison 


\section{Introduction}

18 The red raspberry, Rubus idaeus, is widely distributed in all temperate regions of Europe, Asia, and North America and has been used as food and medicine since $4^{\text {th }}$ century AD (Graham et al., 2004). Often dubbed "European red raspberry", Rubus idaeus is a globally commercialized specialty fruit crop with a large number of commercial varieties, high price, and increasing consumer demands. Owing to its health promoting value, unique flavor, and attractive appearance, Rubus idaeus sales have recently climbed by $8.4 \%$ with world production over 795 , 000 tons (Darnell et al., 2006)(Barney et al., 2007; Food and Agriculture Organization of the United Nations Statistics Division (FAOSTAT)). In addition to its economic and healthpromoting value, the red raspberry plants possess interesting and sometimes unique biological characteristics such as cold hardiness, aggregate fruits, perennial roots and biennial canes, either summer-bearing or ever-bearing flowering/fruiting, and large numbers of hybrids and cultivars. However, red raspberry breeding and research has fallen behind relative to other special fruit crops due to poor seed germination, an absence of reference genome, and limited transcriptome data (Graham and Woodhead, 2009; Hyun et al., 2014). With the recent publication of a high quality black raspberry (Rubus occidentalis) genome (VanBuren et al., 2018), this red raspberry genome allows comparative genomics, genetic breeding, and gene identification of this globally commercialized berry.

Rubus idaeus is a member of the economically important Rosaceae family that also includes rose, peach, apple, cherry, pear, almond, strawberry, and blackberry. Up to now, the genomes of several Rosaceae family members have been sequenced, including Rubus occidentalis (black raspberry) (VanBuren et al., 2016, 2018), Malus x domestica (apple) (Daccord et al., 2017; Velasco et al., 2010), Prunus persica (peach) (Ahmad et al., 2011; Verde et al., 2013), Pyrus

41 bretschneideri (Chinese pear) and Pyrus communi (Chagne et al., 2014) (Chagné et al., 2014),

42 Fragaria vesca (woodland strawberry) (Edger et al., 2018; Shulaev et al., 2011), Potentilla

43 micranthia (mock strawberry) (Buti et al., 2018), and Rosa chinensis (Chinese rose) (Hibrand

44 Saint-Oyant et al., 2018; Raymond et al., 2018). Due to the small genome size, wide variety of

45 fruit types (pomes, drupes, achenes, hips, follicles and capsules), and plant growth habits

46 (ranging from herbaceous to cane, bush and tree forms), Rosaceous genomes offer one of the

47 best systems for the comparative studies in genome evolution and development (Xiang et al., 
2017). The availability of whole-genome sequences of key diploid species such as Rubus idaeus in this family will be crucial to these efforts.

Here, we report a draft genome assembly of red raspberry, Rubus idaeus (Joan J. variety), using long reads of single-molecular real-time (SMRT) Pacific Biosciences sequencing as well as high coverage Illumina short reads. The resulting draft genome is $300 \mathrm{Mbp}$ in size with a BUSCO$\mathrm{Kb}$. Using RNA-seq data from dissected fruit tissues at two developmental stages, we annotated the genome yielding 35,566 protein coding genes with a BUSCO-calculated transcriptome completeness score of $97.2 \%$. We anchored the genome to two previously published high density linkage maps of Rubus idaeus (Ward et al., 2013), facilitating future marker development, breeding, and identification of genes controlling useful trait characteristics. Future comparative analysis, bolstered by this reference sequence, will enable the study of the complex evolution of morphological diversity in fleshy fruits of Rosaceae.

\section{Materials and methods}

\section{Plant material and DNA sequencing}

65 Joan J., a high-yielding, thornless, early primocane raspberry variety was chosen for genome

66 sequencing. The Joan J. variety of Rubus idaeus was obtained from Appalachian Fruit Research

67 Station of USDA ARS. Its genomic DNA was extracted from young leaves using the

68 NucleoSpin® $\square$ Plant $\square$ II $\square$ Midi kit (MACHEREY-NAGEL, Düren, Germany). DNA was

69 sequenced at the Genomics Resource Center of the University of Maryland School of Medicine's

70 Institute of Genome Sciences. Specifically, a long read (5-20kb) PacBio genomic library was

71 constructed using SMRTBell Template Prep Kit and sequenced on two SMRT cells on the

72 PacBio Sequel System, generating 1,305,619 sequence reads with an average length of 9,879 bp

73 (Supplementary Table 1). At the same time, a DNA-seq library was constructed using TruSeq

74 DNA Library Pre Kits (Illumina) and then sequenced on Illumina HiSeq4000 platform in a single

75 lane, yielding 249,081,860 reads of PE150 (Supplementary Table 1). 
PCR adapter sequences were removed using cutadapt (Martin, 2013). Jellyfish (Marçais and Kingsford, 2011) was then used to perform the k-mer distribution analysis with $\mathrm{k}=31$ (Supplementary Figure 1).

\section{Genome Assembly}

83 The genome assembly pipeline is shown in Supplementary Figure 2. A mixture of Illumina short

84 reads and Pacbio long reads (Supplemental Table 1) were assembled into contigs using

85 MaSuRCA; an assembler which combines the efficiency of de Bruijn graph and Overlap-Layout-

86 Consensus approaches (Zimin et al., 2013). The specific settings used in the configuration file

87 other than default were PE $=$ pe 180 20, JF_SIZE $=200000000$ and SOAP_ASSEMBLY=0.

88 Subsequently, Redundans was used to remove heterozygous contigs using an all versus all BLAT

89 approach (Pryszcz and Gabaldón, 2016). The Redundans pipeline also performed scaffolding

90 using a mixture of Illumina short reads and Pacbio long reads (Pryszcz and Gabaldón, 2016).

91 The genomes of Potentilla micranthia (Buti et al., 2018), Rubus occidentalis (VanBuren et al.,

92 2016), and Fragaria vesca (Edger et al., 2018) were leveraged to improve scaffolding using

93 MeDuSa (Bosi et al., 2015). Scaffolds with less than 10X coverage were removed and scaffolds

94 with more than 500 consecutive N's were split. Bowtie2 version 2.3.0 (Langmead and Salzberg,

95 2012) was used to map the Illumina reads back onto the genome prior to Pilon with maximum

96 fragment length to be 1000 and default settings otherwise. The mapping rate was $97.8 \%$ which

97 further validates assembly quality. Pilon (Walker et al., 2014) was then used for one iteration to

98 correct bases, fix misassembly and fill assembly gaps using the diploid parameter. Repeats were

99 then softmasked by first creating a custom repeat library with RepeatModeler -1.0.11

100 (http://www.repeatmasker.org/RepeatModeler/) using the NCBI engine option and then using

101 RepeatMasker (http://www.repeatmasker.org). Lastly, Haplomerger2 (Huang et al., 2017) split

102 the resulting assembly into two sub-assemblies to further remove hetereozygosity.

\section{Sample collection and RNA-sequencing}

105 Raspberry fruit from the Joan J. variety was dissected and separated into three tissues: ovary

106 wall, seed (or ovule), and receptacle. The fruit was collected at two developmental stages, 0 and

10712 DPA. Three biological replicates for above 6 tissues were obtained (Supplementary Table 2).

108 Each tissue was homogenized in the presence of liquid nitrogen. Total RNA extraction was 
109 performed following a previously published protocol (Jones et al., 1997) with few modifications.

110 The CTAB solution (3\% CTAB, 100 mM Tris-HCl pH 8.0, 1.5 M NaCl, 20 mM EDTA,

$1115 \% \mathrm{PVP}$, and $1 \% \beta$-mercaptoethanol made just before use) was added. $10 \mathrm{M}$ Licl solution was

112 mixed with total RNA for two days to precipitate RNA. The total RNA samples were eluted in

113 DEPC-treated $\mathrm{H}_{2} \mathrm{O}$ and stored in $-80{ }^{\circ} \mathrm{C}$.

115 Total RNA was shipped to the Weill Cornell's Genomics and Epigenemics Core Facility, where

116 polyA was isolated and RNA-seq libraries made using Tru-Seq RNA Library Prep Kit.

117 Subsequently, the RNA-seq libraries were sequenced on Illumina HiSeq4000, yielding a total of

$1181,057,377,357$ reads; an average of $96.24 \%$ of these reads mapped to the genome

119 (Supplementary Table 2).

\section{Genome annotation}

123 Repeat Masker was used with a custom repeat library built with Repeat Modeler to soft-mask the

124 genome, and then a combination of $a b$ initio and alignment guided assembly was employed to

125 annotate the soft-masked genome. The Illumina Reads of RNA-seq data described above were

126 trimmed with Trimmomatic (Bolger et al., 2014). RNA-Seq reads were mapped onto the draft

127 genome sequence using Bowtie2 (Langmead et al., 2009). The bam file obtained was used to

128 generate the training set for the gene prediction of BRAKER1 pipeline (Hoff et al., 2016).

129 Candidate transcripts containing no known protein domains by Interproscan5 (Jones et al., 2014)

130 were removed from the final set (13.96\% percent decrease).

132 Trinity was then used to assemble the transcriptome on both genome guided and de novo settings

133 (Grabherr et al., 2011). Prior to trinity assembly, reads were normalized using the perl script

134 provided by Trinity and aligned using Bowtie2 (Grabherr et al., 2011; Langmead et al., 2009).

135 Trinity assemblies were amassed into a comprehensive transcriptome database using PASA

136 (Haas et al., 2003). Lastly, cd-hit-v4.6.8 (Li and Godzik, 2006) was used to cluster transcriptome

137 assemblies from the resulting PASA and BRAKER1 assemblies with over 95\% identity into

138 unigenes. Unigenes that did not map to the genome, had no RNA-seq evidence, and had no

139 known protein domains or orthologues were removed. 
141 Blast2GOPro version 5.1.1 was used to associate Gene Ontology (GO) terms to the resulting

142 transcripts (Supplementary Data 1). Protein sequences were searched against the non-redundant

143 (nr) database protein database from NCBI using BLASTP with an e-value cutoff of 1.0E-3

144 (Conesa et al., 2005). InterProScan was run using default databases in order to assign putative

145 domains to each transcript.

GO enrichment

148 GO enrichment tests were performed to understand potential function of Rubus specific genes.

149 GO term enrichment p-values were calculated using the Fisher's exact test in the TopGO R

150 package (http://bioconductor.org/packages/release/bioc/html/topGO.html). P-values were then

151 adjusted using R's FDR method.

\section{Anchorage to linkage maps}

154 BLAT was run with default settings to identify unique and complete matches to each marker

155 (Kent, 2002). After preparing the input files from BLAT (Supplementary Data 2),

156 pseudochromosomes were then constructed using ALLMAPS with default parameters (Tang et

157 al., 2015). Each genetic map was given a weight of 1. Chimeric scaffolds were manually broken

158 at positions with low coverage, correcting many misassemblies. The seven pseudochromosomes

159 were then constructed by integrating $98 \%$ of the markers from the genetic map.

\section{Comparative genomics}

162 Orthology was established using OrthoFinder-1.1.2 (Emms and Kelly, 2015) using default

163 parameters to infer a rooted species tree and identify orthologous gene groups. Subsequent to the

164 gene trees Orthofinder also produced the species tree. The resulting orthogroups and species tree

165 were then visualized with UpSetR (Conway et al., 2017) and an adjacent phylogenetic tree

166 visualized with iTOL (Letunic and Bork, 2016) (Figure 2A). A Circos plot (Krzywinski et al.,

167 2009) was created by creating links between every gene pair determined to be orthologs (Figure

168 2B-D). Syntenic orthologues were established by using MCScanX (Wang et al., 2012) with

169 settings -s 5. An all by all BLASTp (Boratyn et al., 2013) query with an e-value cutoff of 1e-10

170 was performed and used as a basis for MCScanX with default parameters to identify syntenic

171 gene regions. 


\section{Results and Discussion}

\section{Genome assembly and annotation}

177 Rubus idaeus is a diploid species $(2 \mathrm{n}=2 \mathrm{x}=14)$ with an estimated genome size of $293 \mathrm{Mbp}$ based on flow cytometry analysis (Graham and Woodhead, 2009). We first sequenced the Rubus idaeus genome using 120X Illumina coverage (Supplementary Table 1). The distribution of kmers indicates that the Rubus idaeus genome is approximately $303 \mathrm{Mbp}$ (Methods), and the bimodal distribution of 31-mers (Supplemental Figure 1) suggests significant polymorphism and heterozygosity in the genome.

To overcome the issue of heterozygosity for genome assembly, a hybrid genome assembly approach was used taking advantage of both the sequencing depth and accuracy offered by the Illumina platform (at $120 \mathrm{X}$ coverage) and the sequence length offered by the PacBio platform (at 26X overage) (Supplemental Table 1). The pipeline of the assembly is outlined in Supplemental Figure 2. We used Redundans (Pryszcz and Gabaldón, 2016) and Haplomerger2 (Huang et al., 2017) tools to correct for heterozygosity. A comparative genomic approach (Bosi et al., 2015; Pop et al., 2004) was used as part of the genome assembly. Specifically, the most recently assembled genomes of closely related species Potentilla micranthia (Buti et al., 2018), Rubus

192 occidentalis (VanBuren et al., 2016), and Fragaria vesca (Edger et al., 2018) were leveraged to

193 improve scaffolding using MeDuSa (Bosi et al., 2015). The resulting $R$. idaeus genome assembly

194 is $300 \mathrm{Mbp}$ in size, containing 2,145 scaffolds with a N50 of $638 \mathrm{~Kb}$ (Table 1). To assess the

195 completeness of the genome, BUSCO v.3.0.2 (Simão et al., 2015) was used to locate the

196 presence or absence of the embryophyta_odb9 (plant) dataset. The BUSCO Completeness Score reached $95.3 \%$ (Table 1 ), which validates the good assembly quality.

199 To annotate the Rubus idaeus genome, a transcriptome was generated from 1,057,377,357

200 Illumina RNA-seq reads pooled from 18 RNA-seq libraries derived from three different fruit 201 tissues (ovary wall, ovule/seed, receptacle) at two developmental stages (0 and 12 Days Post202 Anthesis or DPA) in three biological replicates (Supplemental Table 2). A combination of $a b$ 203 initio and alignment guided assembly was employed to annotate the genome (soft-masked for 
204 repeats). This resulted in 35,566 protein coding genes with a BUSCO-calculated transcriptome 205 completeness score of $97.2 \%$ (Table 1). The high completeness score indicates that transcripts 206 from almost all genes expressed in these tissues have been sequenced. Finally, Blast2GO was 207 used to associate Gene Ontology (GO) terms to the annotated genes (Supplementary Data 1).

\section{Anchoring scaffolds to genetic maps}

210 The scaffolds were anchored onto pseudochromosomes (Figure 1) taking advantage of two 211 previous genetic linkage maps. They are respectively the 'Heritage' and 'Tulameen' variety-

212 based linkage maps that collectively contained 4225 markers. As a result, the

213 pseudochromosomes contain $80.1 \%$ of the assembly (ie. at $240 \mathrm{Mb}$ ). The average magnitude of

214 the Pearson correlation coefficient between the physical and map locations is 0.92 showing a

215 high consistency between the genome and previously published linkage maps (Figure 1;

216 Supplementary Data 2).

\section{Comparative Genomics}

219 Orthologous gene groups were established from 10 angiosperms using OrthoFinder-1.1.2 (Emms

220 and Kelly, 2015); these include 9 members of the Rosaceae family (Prunus persica, Pyrus

221 communis, Malus x domestica, Rosa chinesis, Rosa multiflora, Rubus occidentalis, Rubus

222 idaeus, Fragaria vesca, Potentilla micrantha) and the model organism Arabidopsis thaliana,

223 used here as an outlier species to root the tree. The resulting phylogenetic tree (Figure 2A) is

224 consistent with previously published phylogenetic analyses of the Rosaceae family (Xiang et al.,

225 2017). In total 25,193 orthogroups were established (Supplementary Data 3). As shown in Figure

226 2A, 10,205 orthogroups contained proteins from all 9 Rosaceae species as well as Arabidopsis.

227 Interestingly, many specific orthogroups $(1,878)$ are unique to Malus x domestica and Pyrus

228 communis. Both species belong to the subfamily Maleae, which has undergone a whole genome

229 duplication, at its origin (Daccord et al., 2017; Wu et al., 2013; Xiang et al., 2017). The large

230 number of orthogroups shared between Malus x domestica and Pyrus communis suggests that

231 substantial diversification occurred after whole genome duplication (WGD) within the Maleae

232 subfamily, which may have contributed to the subfamily's pome fruit type (Velasco et al., 2010;

233 Xiang et al., 2017). Expectedly, all members of the Rosaceae family share many orthogroups

$234(1,420)$ that are distinct from Arabidopsis thaliana. Members of the same genus also show a high 
235 number of common gene families. Specifically, there are 1,071 and 775 orthogroups limited to

236 the Rosa and Rubus genera, respectively (Figure 2A, Supplementary Data 3). As Rubus is one of

237 the largest and most morphologically diverse genus in the Rosaceae family (Alice and Campbell,

238 1999), we examined GO term enrichment among the 775 Rubus-specific orthogroups

239 (Supplementary Data 4). Significantly enriched GO terms include chromatin assembly, RNA-

240 splicing, and fungal-type cell wall organization, suggesting that Rubus-specific genes are

241 involved in gene regulation and defense.

243 Strawberry and raspberry share the same base chromosome number $(n=7)$, with estimated

244 divergence time of 75 million years (Xiang et al., 2017). Rubus occidentalis and Rubus idaeus,

245 on the other hand, are closely related species. Syntenic blocks revealed a high collinearity

246 between Rubus idaeus and Rubus occidentalis and between Rubus idaeus and F. vesca (Figure

247 2B and C). R. occidentalis had 25,289 gene pairs represented within 1,596 collinear blocks with

$248 R$. idaeus. F. vesca and $R$. idaeus shared 17,769 syntenic gene pairs within 887 collinear blocks.

249 This high degree of synteny helps validate the Rubus idaeus assembly. When compared with the

250 more distant peach genome, Prunus persica, which has a different base chromosome number

251 (n=8), collinearity decreases slightly: P. persica and R. idaeus share 17,064 gene pairs on 877

252 collinear regions. Although there is lower collinearity, there are strikingly large conserved

253 syntenic blocks. For example, a large portion of $R$. idaeus chromosome 7 is syntenic to $P$.

254 persica chromosome 2 while a smaller portion of $R$. idaeus chromosome 7 syntenic to $P$. persica

2557 (Figure 2D).

257 To facilitate future functional studies of raspberry development, the Rubus idaeus genome

258 assembly version 1 file, total transcript version 1 file, and annotation version 1 gff3 file are

259 provided as Supplementary Data 5, 6, and 7 respectively. The Transcription Factors (TFs) and

260 major hormonal pathway genes of $R$. idaeus are also identified and provided as Supplementary

261 Data 8. Together with the GO assignment (Supplementary Data 1), linkage between physical and

262 genetic markers (Supplementary Data 2), and ortholog assignment of nine Rosaceae species

263 (Supplementary Data 3), these new genomic resources will assist raspberry research and

264 breeding. 


\section{Supplemental Information}

268 Supplementary Table 1. Summary statistics of DNA sequence data for Rubus idaeus genome 269 assembly

270 Supplementary Table 2. Summary statistics of RNA-seq data for Rubus idaeus fruit tissues.

271 Supplementary Figure 1. Bimodal K-mer distribution of Rubus idaeus (variety Joan J.) genome.

272 Supplemental Figure 2. Genome assembly pipeline.

273 Supplementary Data 1: GO annotations associated with Rubus idaeus transcripts

274 Supplementary Data 2: Correlation between scaffold positions and genetic markers

275 Supplementary Data 3: Orthology clustering of Rosaceae species and Arabidopsis

276 Supplementary Data 4: GO enrichment of Rubus-specific genes

277 Supplementary Data 5: Rubus idaeus_genome_v1.fa.gz

278 Supplementary Data 6: Rubus idaeus_transcript_v1.fa.gz

279 Supplementary Data 7: Rubus idaeus_annotation_v1.gff3

280 Supplementary Data 8: Orthologs of known Arabidopsis transcription factors and hormone

281 related genes

\section{Funding}

284 H.W. is supported by the NSF Computation and Mathematics for Biological Networks Research

285 Traineeship (NSF_NRT 1632976). The work is supported by NSF grants (IOS1444987) to S.H. 286 and Z.L.

288 Conflict of interest: None declared.

290 Availability of supporting data

291 The genomic DNA-sequencing and RNA-sequencing data supporting the results of this article

292 are available at Sequence Read Archive of NCBI with accession numbers SRP4284044 and

293 SRP153061 respectively. 
bioRxiv preprint doi: https://doi.org/10.1101/546135; this version posted February 18,2019 . The copyright holder for this preprint (which was not certified by peer review) is the author/funder, who has granted bioRxiv a license to display the preprint in perpetuity. It is made available under aCC-BY-NC-ND 4.0 International license.

11

296 We would like to thank Drs. Ann Callahan and Chris Dardick at USDA ARS for the Joan J.

297 Rubus idaeus plants and Miss Anuhyea Pulapaka for help with the genome assembly. 


\section{References}

Ahmad, R., Parfitt, D.E., Fass, J., Ogundiwin, E., Dhingra, A., Gradziel, T.M., Lin, D., Joshi, N.A., Martinez-Garcia, P.J., and Crisosto, C.H. (2011). Whole genome sequencing of peach (Prunus persica L.) for SNP identification and selection. BMC Genomics 12, 569.

Alice, L.A., and Campbell, C.S. (1999). Phylogeny of Rubus (rosaceae) based on nuclear ribosomal DNA internal transcribed spacer region sequences. Am. J. Bot. 86, 81-97.

Barney, D.L., Bristow, P., Cogger, C., Fitzpatrick, S.M., Hart, J., Kaufman, D., Miles, C., Miller, T., Moore, P.P., Murray, T. and Rempel, H. (2007). Commercial red raspberry production in the Pacific Northwest. Pacific Northwest Ext. Publ. PNW, 598.

Bolger, A.M., Lohse, M., and Usadel, B. (2014). Trimmomatic: a flexible trimmer for Illumina sequence data. Bioinformatics (Oxford, England) 30, 2114-2120.

Boratyn, G.M., Camacho, C., Cooper, P.S., Coulouris, G., Fong, A., Ma, N., Madden, T.L., Matten, W.T., McGinnis, S.D., Merezhuk, Y., et al. (2013). BLAST: a more efficient report with usability improvements. Nucleic Acids Res 41, W29-W33.

Bosi, E., Donati, B., Galardini, M., Brunetti, S., Sagot, M.-F., Lió, P., Crescenzi, P., Fani, R., and Fondi, M. (2015). MeDuSa: a multi-draft based scaffolder. Bioinformatics 31, 2443-2451.

Buti, M., Moretto, M., Barghini, E., Mascagni, F., Natali, L., Brilli, M., Lomsadze, A., Sonego, P., Giongo, L., Alonge, M. and Velasco, R., (2018). The genome sequence and transcriptome of Potentilla micrantha and their comparison to Fragaria vesca (the woodland strawberry). GigaScience, 7(4), pp.1-14.

Chagné, D., Crowhurst, R.N., Pindo, M., Thrimawithana, A., Deng, C., Ireland, H., Fiers, M., Dzierzon, H., Cestaro, A., Fontana, P., et al. (2014). The Draft Genome Sequence of European Pear (Pyrus communis L. "Bartlett"). PLoS ONE 9, e92644-e92644.

Conesa, A., Gotz, S., Garcia-Gomez, J.M., Terol, J., Talon, M., and Robles, M. (2005). Blast2GO: a universal tool for annotation, visualization and analysis in functional genomics research. Bioinformatics 21, 3674-3676.

Conway, J.R., Lex, A., and Gehlenborg, N. (2017). UpSetR: an R package for the visualization of intersecting sets and their properties. Bioinformatics 33, 2938-2940.

Daccord, N., Celton, J.-M., Linsmith, G., Becker, C., Choisne, N., Schijlen, E., van de Geest, H., Bianco, L., Micheletti, D., Velasco, R., et al. (2017). High-quality de novo assembly of the apple genome and methylome dynamics of early fruit development. Nature Genetics 49, 1099-1106.

Darnell, R.L., Alvarado, H.E., Williamson, J.G., Brunner, B., Plaza, M., and Negrón, E. (2006). Annual, Off-season Raspberry Production in Warm Season Climates. HortTechnology 16, 9297.

Edger, P.P., VanBuren, R., Colle, M., Poorten, T.J., Wai, C.M., Niederhuth, C.E., Alger, E.I., Ou, S., Acharya, C.B., Wang, J., et al. (2018). Single-molecule sequencing and optical mapping 
yields an improved genome of woodland strawberry (Fragaria vesca) with chromosome-scale contiguity. GigaScience 7, 1-7.

Emms, D.M., and Kelly, S. (2015). OrthoFinder: solving fundamental biases in whole genome comparisons dramatically improves orthogroup inference accuracy. Genome Biology 16, 157.

Grabherr, M.G., Haas, B.J., Yassour, M., Levin, J.Z., Thompson, D.A., Amit, I., Adiconis, X., Fan, L., Raychowdhury, R., Zeng, Q., et al. (2011). Full-length transcriptome assembly from RNA-Seq data without a reference genome. Nature Biotechnology 29, 644-652.

Graham J., Woodhead M. (2009) Raspberries and Blackberries: The Genomics of Rubus. In: Folta K.M., Gardiner S.E. (eds) Genetics and Genomics of Rosaceae. Plant Genetics and Genomics: Crops and Models, vol 6. Springer, New York, NY

Graham, J., Smith, K., MacKenzie, K., Jorgenson, L., Hackett, C., and Powell, W. (2004). The construction of a genetic linkage map of red raspberry (Rubus idaeus subsp. idaeus) based on AFLPs, genomic-SSR and EST-SSR markers. Theor Appl Genet 109, 740-749.

Haas, B.J., Delcher, A.L., Mount, S.M., Wortman, J.R., Smith, R.K., Hannick, L.I., Maiti, R., Ronning, C.M., Rusch, D.B., Town, C.D., et al. (2003). Improving the Arabidopsis genome annotation using maximal transcript alignment assemblies. Nucleic Acids Research 31, 5654 5666.

Hibrand Saint-Oyant, L., Ruttink, T., Hamama, L., Kirov, I., Lakhwani, D., Zhou, N.N., Bourke, P.M., Daccord, N., Leus, L., Schulz, D., et al. (2018). A high-quality genome sequence of Rosa chinensis to elucidate ornamental traits. Nature Plants 4, 473-484.

Hoff, K.J., Lange, S., Lomsadze, A., Borodovsky, M., and Stanke, M. (2016). BRAKER1: Unsupervised RNA-Seq-Based Genome Annotation with GeneMark-ET and AUGUSTUS: Table 1. Bioinformatics 32, 767-769.

Huang, S., Kang, M., and Xu, A. (2017). HaploMerger2: rebuilding both haploid sub-assemblies from high-heterozygosity diploid genome assembly. Bioinformatics 33, 2577-2579.

Hyun, T.K., Lee, S., Rim, Y., Kumar, R., Han, X., Lee, S.Y., Lee, C.H., and Kim, J.-Y. (2014). De-novo RNA Sequencing and Metabolite Profiling to Identify Genes Involved in Anthocyanin Biosynthesis in Korean Black Raspberry (Rubus coreanus Miquel). PLoS One 9(2), e88292.

Jones, C.S., Iannetta, P.P.M., Woodhead, M., Davies, H.V., McNicol, R.J., and Taylor, M.A. (1997). The isolation of RNA from raspberry (Rubus idaeus) fruit. Molecular Biotechnology 8 , 219-221.

Jones, P., Binns, D., Chang, H.-Y., Fraser, M., Li, W., McAnulla, C., McWilliam, H., Maslen, J., Mitchell, A., Nuka, G., et al. (2014). InterProScan 5: genome-scale protein function classification. Bioinformatics (Oxford, England) 30, 1236-1240.

Kent, W.J. (2002). BLAT--the BLAST-like alignment tool. Genome Research 12, 656-664. 
Krzywinski, M., Schein, J., Birol, I., Connors, J., Gascoyne, R., Horsman, D., Jones, S.J., and Marra, M.A. (2009). Circos: an information aesthetic for comparative genomics. Genome Research 19, 1639-1645.

Langmead, B., and Salzberg, S.L. (2012). Fast gapped-read alignment with Bowtie 2. Nature Methods 9, 357-359.

Langmead, B., Trapnell, C., Pop, M., and Salzberg, S.L. (2009). Ultrafast and memory-efficient alignment of short DNA sequences to the human genome. Genome Biology 10, R25.

Letunic, I., and Bork, P. (2016). Interactive tree of life (iTOL) v3: an online tool for the display and annotation of phylogenetic and other trees. Nucleic Acids Res. 44, W242-245.

Li, W., and Godzik, A. (2006). Cd-hit: a fast program for clustering and comparing large sets of protein or nucleotide sequences. Bioinformatics 22, 1658-1659.

Marçais, G., and Kingsford, C. (2011). A fast, lock-free approach for efficient parallel counting of occurrences of k-mers. Bioinformatics (Oxford, England) 27, 764-770.

Martin, M. (2013). Cutadapt removes adapter sequences from high-throughput sequencing reads. EMBnet.journal 17, 10-12.

Pop, M., Kosack, D.S., and Salzberg, S.L. (2004). Hierarchical scaffolding with Bambus. Genome Research 14, 149-159.

Pryszcz, L.P., and Gabaldón, T. (2016). Redundans: an assembly pipeline for highly heterozygous genomes. Nucleic Acids Research 44, e113.

Raymond, O., Gouzy, J., Just, J., Badouin, H., Verdenaud, M., Lemainque, A., Vergne, P., Moja, S., Choisne, N., Pont, C., et al. (2018). The Rosa genome provides new insights into the domestication of modern roses. Nature Genetics 50, 772-777.

Shulaev, V., Sargent, D.J., Crowhurst, R.N., Mockler, T.C., Folkerts, O., Delcher, A.L., Jaiswal, P., Mockaitis, K., Liston, A., Mane, S.P., et al. (2011). The genome of woodland strawberry (Fragaria vesca). Nature Genetics 43, 109-116.

Simão, F.A., Waterhouse, R.M., Ioannidis, P., Kriventseva, E. V., and Zdobnov, E.M. (2015). BUSCO: assessing genome assembly and annotation completeness with single-copy orthologs. Bioinformatics 31, 3210-3212.

Tang, H., Zhang, X., Miao, C., Zhang, J., Ming, R., Schnable, J.C., Schnable, P.S., Lyons, E., and Lu, J. (2015). ALLMAPS: robust scaffold ordering based on multiple maps. Genome Biology 16, 3.

VanBuren, R., Bryant, D., Bushakra, J.M., Vining, K.J., Edger, P.P., Rowley, E.R., Priest, H.D., Michael, T.P., Lyons, E., Filichkin, S.A., et al. (2016). The genome of black raspberry ( Rubus occidentalis ). The Plant Journal 87, 535-547. 
VanBuren, R., Wai, C.M., Colle, M., Wang, J., Sullivan, S., Bushakra, J.M., Liachko, I., Vining, K.J., Dossett, M., Finn, C.E., et al. (2018). A near complete, chromosome-scale assembly of the black raspberry (Rubus occidentalis) genome. Gigascience 7.

Velasco, R., Zharkikh, A., Affourtit, J., Dhingra, A., Cestaro, A., Kalyanaraman, A., Fontana, P., Bhatnagar, S.K., Troggio, M., Pruss, D., et al. (2010). The genome of the domesticated apple (Malus $\times$ domestica Borkh.). Nature Genetics 42, 833-839.

Verde, I., Abbott, A.G., Scalabrin, S., Jung, S., Shu, S., Marroni, F., Zhebentyayeva, T., Dettori, M.T., Grimwood, J., Cattonaro, F., et al. (2013). The high-quality draft genome of peach (Prunus persica) identifies unique patterns of genetic diversity, domestication and genome evolution. Nature Genetics 45, 487-494.

Walker, B.J., Abeel, T., Shea, T., Priest, M., Abouelliel, A., Sakthikumar, S., Cuomo, C.A., Zeng, Q., Wortman, J., Young, S.K., et al. (2014). Pilon: An Integrated Tool for Comprehensive Microbial Variant Detection and Genome Assembly Improvement. PLoS ONE 9, e112963.

Wang, Y., Tang, H., DeBarry, J.D., Tan, X., Li, J., Wang, X., Lee, T., Jin, H., Marler, B., Guo, H., et al. (2012). MCScanX: a toolkit for detection and evolutionary analysis of gene synteny and collinearity. Nucleic Acids Res 40, e49.

Ward, J.A., Bhangoo, J., Fernández-Fernández, F., Moore, P., Swanson, J., Viola, R., Velasco, R., Bassil, N., Weber, C.A., and Sargent, D.J. (2013). Saturated linkage map construction in Rubus idaeus using genotyping by sequencing and genome-independent imputation. BMC Genomics 14, 2.

Wu, J., Wang, Z., Shi, Z., Zhang, S., Ming, R., Zhu, S., Khan, M.A., Tao, S., Korban, S.S., Wang, H., et al. (2013). The genome of the pear (Pyrus bretschneideri Rehd.). Genome Research 23, 396-408.

Xiang, Y., Huang, C.-H., Hu, Y., Wen, J., Li, S., Yi, T., Chen, H., Xiang, J., and Ma, H. (2017). Evolution of Rosaceae Fruit Types Based on Nuclear Phylogeny in the Context of Geological Times and Genome Duplication. Molecular Biology and Evolution 34, 262-281.

Zimin, A. V., Marçais, G., Puiu, D., Roberts, M., Salzberg, S.L., and Yorke, J.A. (2013). The MaSuRCA genome assembler. Bioinformatics 29, 2669-2677. 
Table 1. Statistics of genome and transcriptome assemblies. Single (S), Duplicated (D), Fragmented (F) and Missing (M) single-copy orthologs are reported alongside the BUSCO completeness score.

\begin{tabular}{|l|l|}
\hline Total length & $300,259,977 \mathrm{bp}$ \\
\hline Scaffold N50 & $638,152 \mathrm{bp}$ \\
\hline Contig N50 & $250,294 \mathrm{bp}$ \\
\hline Smallest Scaffold & $501 \mathrm{bp}$ \\
\hline Largest Scaffold & $4,458,320 \mathrm{bp}$ \\
\hline N's & $174,429 \mathrm{bp}(.000582 \%)$ \\
\hline Sequence GC's & $37.9 \%$ \\
\hline$\%$ Repeats & $43.35 \%$ \\
\hline Busco Completeness Score (Genome) & $95.3 \%$ (S:86.1\%, D:9.2\%), F:1.5\%, M:3.2\% \\
\hline Number of Annotated Protein Coding Genes & 35,566 \\
\hline Busco Completeness Score (Transcriptome) & $97.2 \%$ (S:92.9\%,D:4.3\%), F:1.1\%, M:1.7\% \\
\hline
\end{tabular}



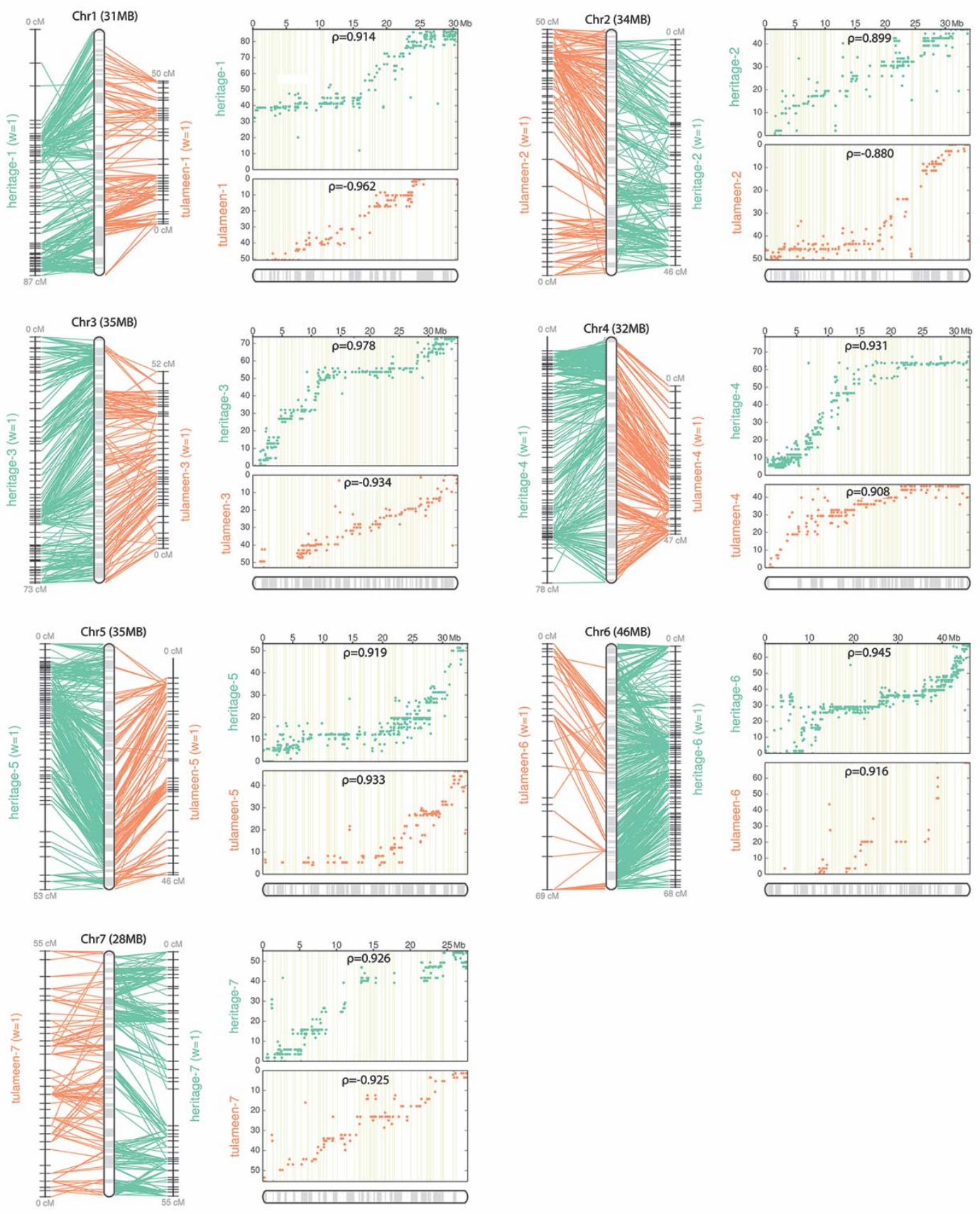

Figure 1. The correlation between physical map and the linkage maps of seven chromosomes.

For each chromosome, the left figure illustrates the connections between physical positions on the assembled pseudomolecule and the two flanking linkage maps colored in orange and teal respectively. The orange coloring represents the tulmaneen linkage map whereas the teal represents the heritage linkage map (Ward et al., 2013). On the right is the scatter plot with dots representing the physical position on the chromosome ( $x$ axis) versus the map position ( $y$ axis). Rho $(\rho)$ is the Pearson correlation coefficient (right panel). Each panel represents distinct chromosome. 
A


Figure 2. The distribution of shared gene families among nine Rosaceae species and Arabidopsis thaliana.

(A)The left panel describes the phylogeny among the species. The branch length distances represent substitutions per site. The right panel is an UpSet plot (Conway et al., 2017): an alternative representation of a venn diagram with intersections (shared genes) greater than 100. The species described in each intersection is represented by the dotted lines, the size of the intersection is described by the bar chart above. (B) Circos plots (Krzywinski et al., 2009) displaying macrosynteny between the genomes of Rubus idaeus and Rubus occidentalis. (C) Macrosynteny between Rubus idaeus and Fragaria vesca. (D) Macrosynteny between Rubus idaeus and Prunus persica. For B to D, each connecting line represents an orthologous gene pair and the right half of each circle consists of the seven Rubus ideaus chromosomes colored by the spectral order in the rainbow. 
bioRxiv preprint doi: https://doi.org/10.1101/546135; this version posted February 18, 2019. The copyright holder for this preprint (which was not certified by peer review) is the author/funder, who has granted bioRxiv a license to display the preprint in perpetuity. It is made available under aCC-BY-NC-ND 4.0 International license.

8

\section{Supplemental Tables}

Supplementary Table 1. Summary statistics of DNA sequence data for Rubus idaeus genome assembly

\begin{tabular}{|l|l|l|l|}
\hline & $\begin{array}{l}\text { Mean Read } \\
\text { length }\end{array}$ & Read count & Total base, bp \\
\hline Illumina PE & 150 & $249,081,860$ & $37,455,877,274$ \\
\hline PacBio & 9,879 & $1,305,619$ & $8,007,129,543$ \\
\hline
\end{tabular}


Supplementary Table 2. Summary statistics of RNA-seq data for Rubus idaeus fruit tissues.

\begin{tabular}{|l|l|l|l|l|}
\hline Sample & $\begin{array}{l}\text { Number of } \\
\text { Reads }\end{array}$ & $\begin{array}{l}\text { \% of Uniquely } \\
\text { Mapped Reads }\end{array}$ & $\begin{array}{l}\text { \% of reads } \\
\text { mapped to } \\
\text { multiple loci }\end{array}$ & $\begin{array}{l}\text { Total \% } \\
\text { reads } \\
\text { mapped }\end{array}$ \\
\hline Ovule-0-26 & 60144925 & $87.94 \%$ & $7.13 \%$ & $95.07 \%$ \\
\hline Ovule-0-41 & 57979785 & $89.56 \%$ & $7.24 \%$ & $96.80 \%$ \\
\hline Ovule-0-7 & 66004490 & $89.29 \%$ & $7.30 \%$ & $96.59 \%$ \\
\hline Receptacle-0-17 & 64293938 & $89.79 \%$ & $7.16 \%$ & $96.95 \%$ \\
\hline Receptacle-0-27 & 61401941 & $89.54 \%$ & $7.18 \%$ & $96.72 \%$ \\
\hline Receptacle-0-41 & 68480278 & $88.20 \%$ & $7.18 \%$ & $95.38 \%$ \\
\hline Receptacle-12-13 & 67769659 & $89.39 \%$ & $6.32 \%$ & $95.71 \%$ \\
\hline Receptacle-12-1 & 54088666 & $91.41 \%$ & $6.22 \%$ & $97.63 \%$ \\
\hline Receptacle-12-4 & 50260693 & $90.69 \%$ & $6.70 \%$ & $97.39 \%$ \\
\hline Seed-12-13 & 53332584 & $84.84 \%$ & $8.07 \%$ & $92.91 \%$ \\
\hline Seed-12-1 & 55781661 & $89.18 \%$ & $8.47 \%$ & $97.65 \%$ \\
\hline Seed-12-7 & 62967294 & $89.73 \%$ & $7.99 \%$ & $97.72 \%$ \\
\hline Wall-0-24 & 65304690 & $89.32 \%$ & $7.27 \%$ & $96.59 \%$ \\
\hline Wall-0-7 & 59200984 & $89.64 \%$ & $7.51 \%$ & $97.15 \%$ \\
\hline Wall-0-13 & 71863354 & $89.68 \%$ & $7.34 \%$ & $97.02 \%$ \\
\hline Wall-12-13 & 68284797 & $83.95 \%$ & $8.43 \%$ & $92.38 \%$ \\
\hline Wall-12-1 & 70217618 & $88.17 \%$ & $8.27 \%$ & $96.44 \%$ \\
\hline Wall-12-4 & 61592260 & $89.07 \%$ & $9.06 \%$ & $98.13 \%$ \\
\hline
\end{tabular}

*Sample names are "Tissue-Stage-unique ID of the specific sample". The two stages are 0 DPA and 12 DPA. The tissues are Ovule, Seed, Receptacle, and Wall (ovary wall). 


\section{Supplemental Figures}

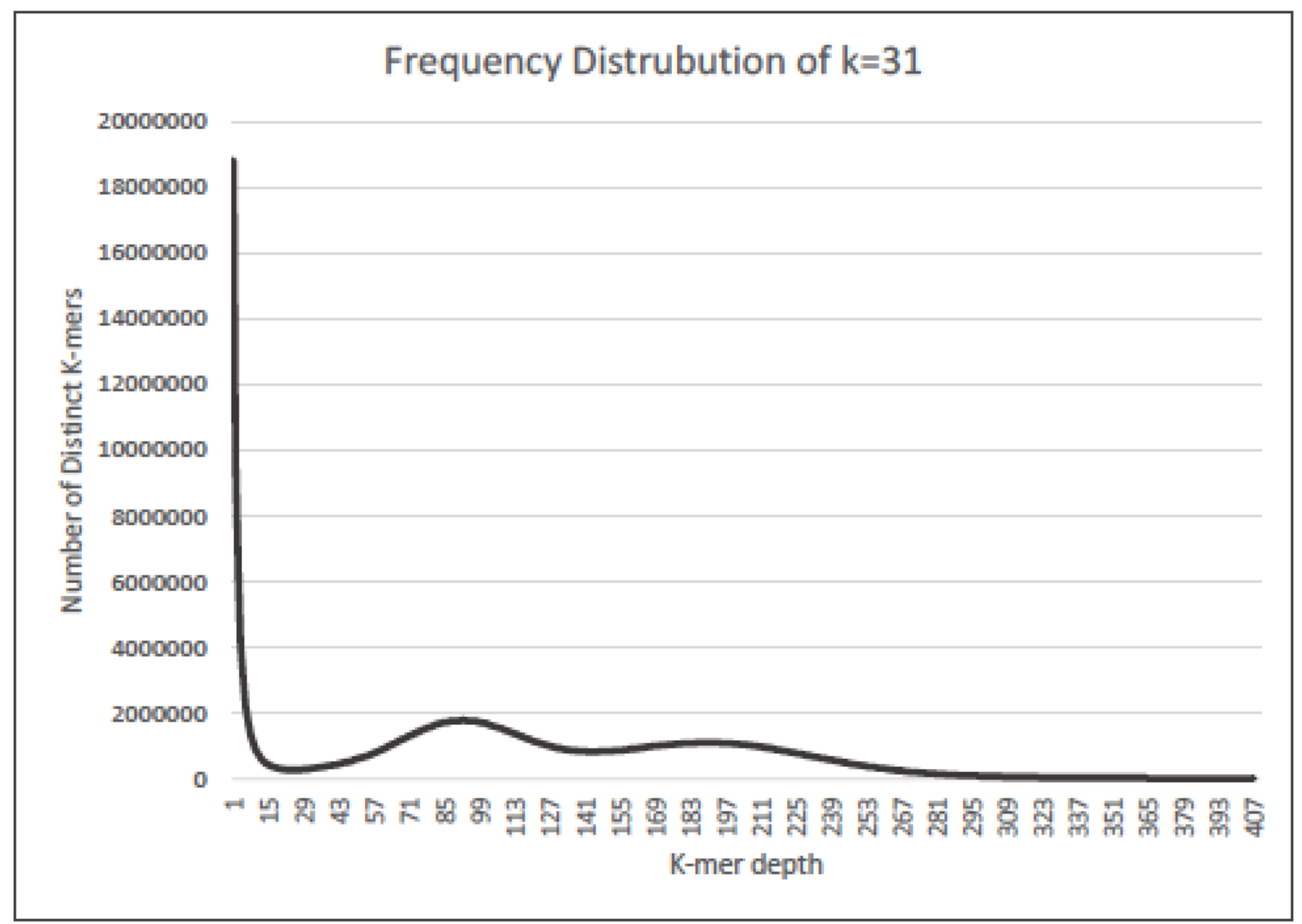

Supplementary Figure 1. Bimodal K-mer distribution of Rubus idaeus (variety Joan J.) genome 31-mer distribution of Rubus idaeus genome obtained, using jellyfish, from 150-bp paired-end whole genome sequencing data. 
bioRxiv preprint doi: https://doi.org/10.1101/546135; this version posted February 18,2019 . The copyright holder for this preprint (which was not certified by peer review) is the author/funder, who has granted bioRxiv a license to display the preprint in perpetuity. It is made available under aCC-BY-NC-ND 4.0 International license.

11

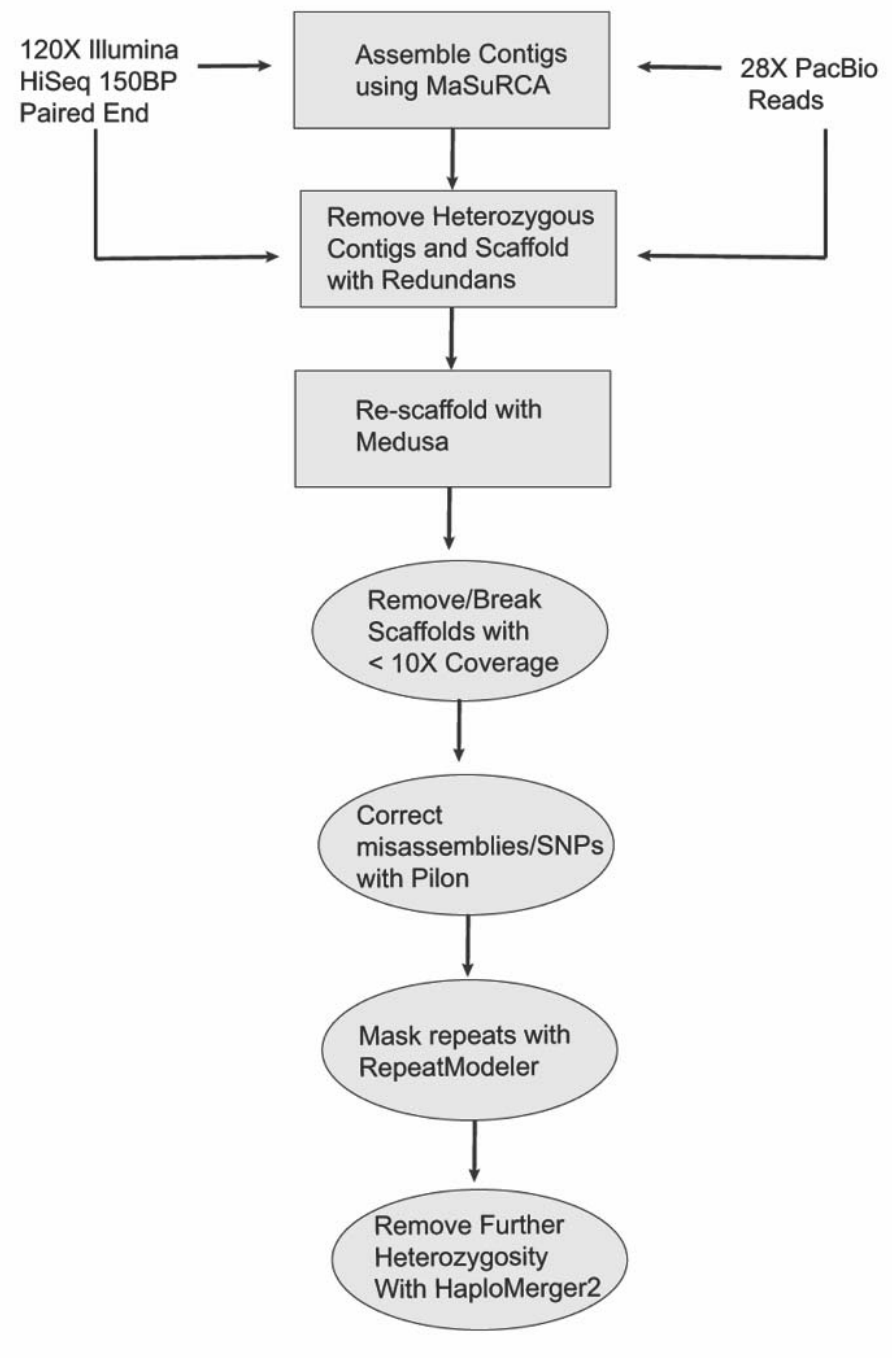

Supplemental Figure 2. Genome assembly pipeline.

Flowchart represents all steps of the genome assembly process upstream of anchoring to the linkage map. 

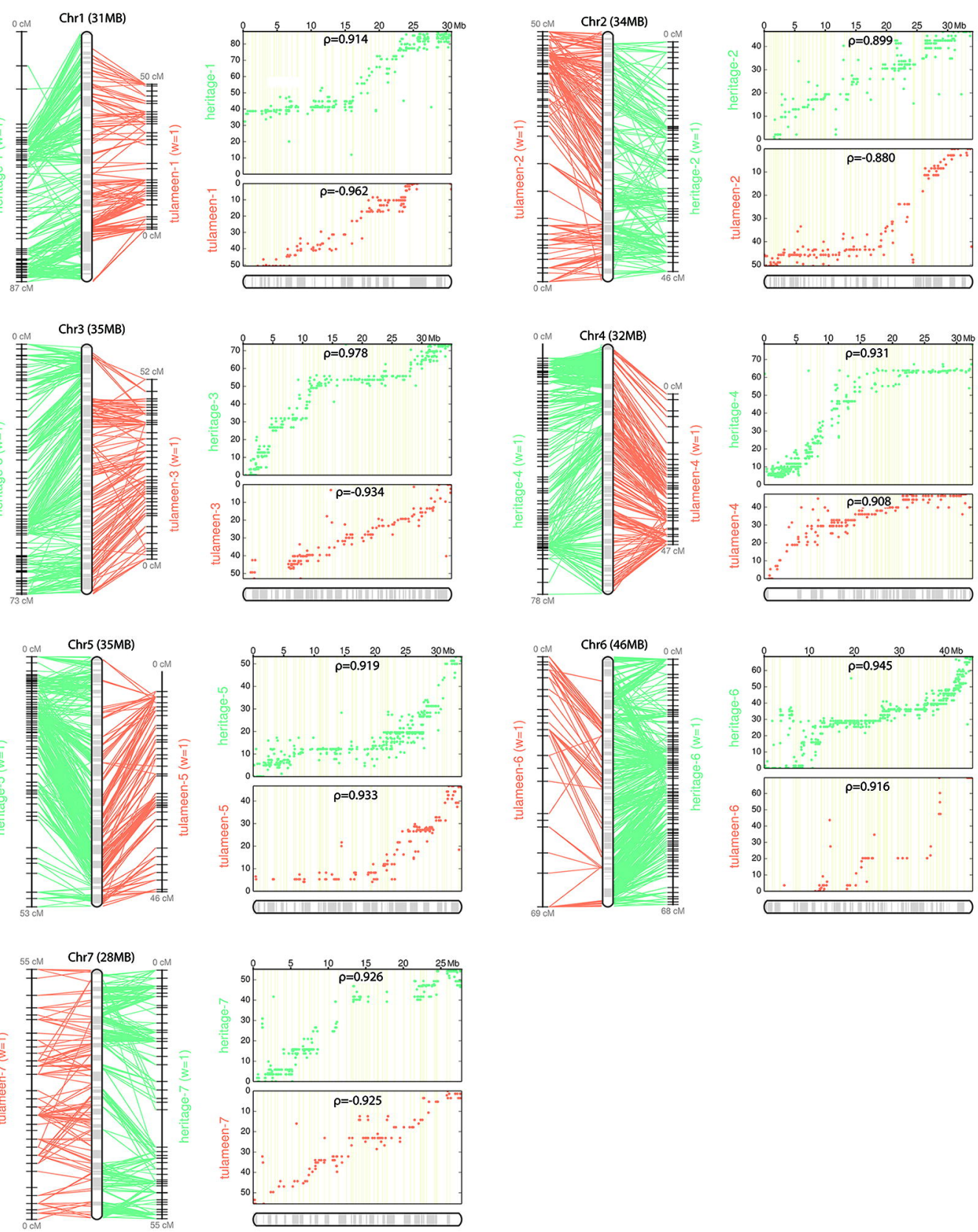
A
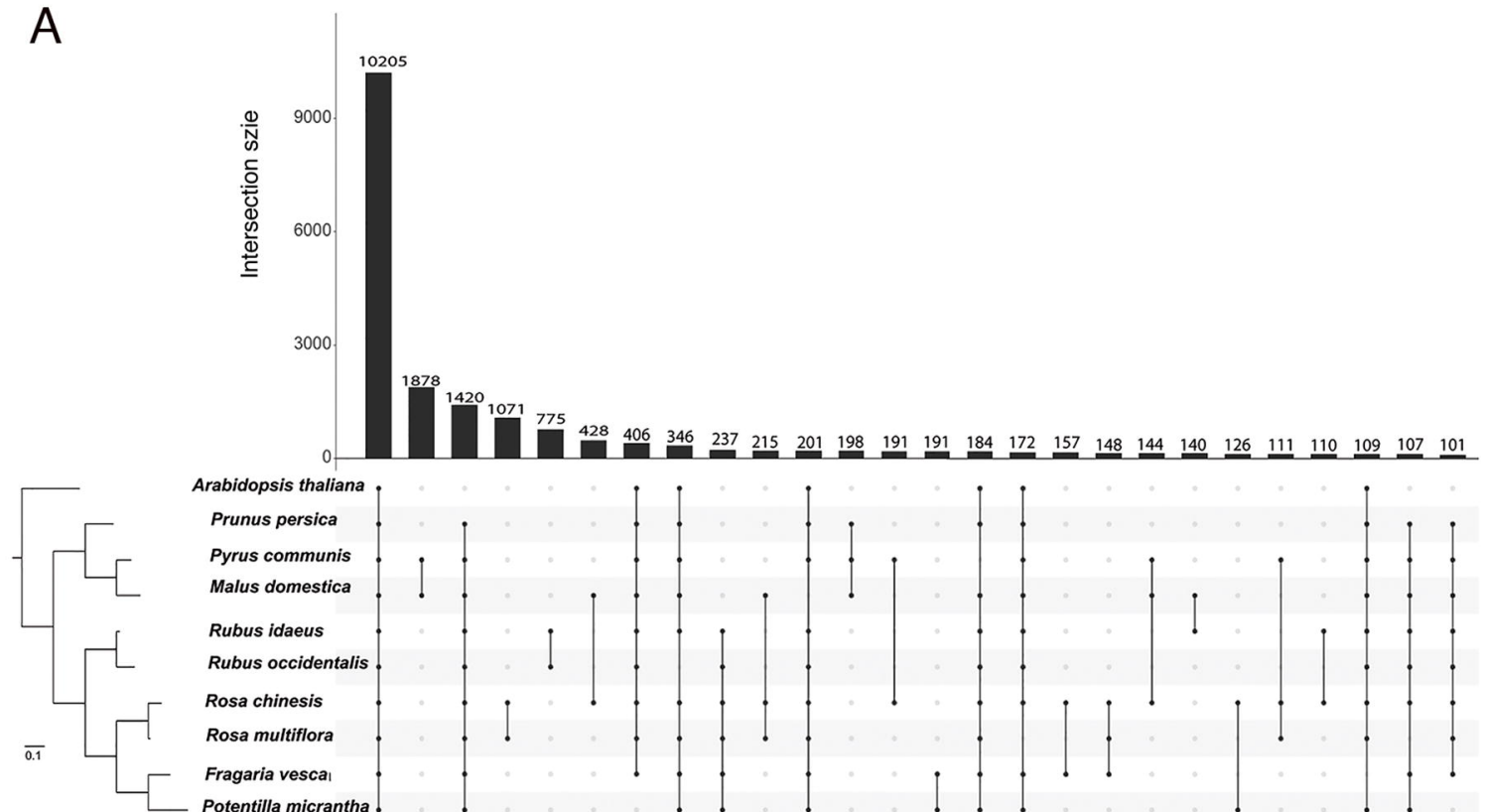

Arabidopsis thaliana Prunus persica

Pyrus communis Malus domestica Rubus idaeus Rubus occidentalis Rosa chinesis Rosa multiflora Fragaria vesca। Potentilla micrantha .
